\title{
Different Methods for Pion-nucleus Optical Potential
}

\author{
S.A.E. Khallaf and A.A. Ibrahim \\ Department of Physics, Assiut University, Assiut 71516, Egypt
}

\begin{abstract}
Elastic and inelastic cross-sections for pion scattering on ${ }^{12} \mathrm{C}$ at pion kinetic energy ranging from 50 to $260 \mathrm{MeV}$ are computed using three independent methods of $\pi^{ \pm}$-nucleus optical potential, the 3 $\alpha$-particle model of the nucleus, the equivalent local Kisslinger potential and the Laplacian one. Reasonable fits to the measured values are obtained for ${ }^{12} \mathrm{C}$ without adjusting free parameters. The ability of these methods to account for elastic, inelastic, total and reaction cross-section data are somewhat similar. The Kisslinger-based local potential is the more suitable for describing the elastic and inelastic cross-sections of $\pi^{ \pm}$-nucleus scattering. It seems that the $3 \alpha$-particle model of ${ }^{12} \mathrm{C}$ is not useful in the description of pion scattering on ${ }^{12} \mathrm{C}$ at least in the $\Delta$-resonance region.
\end{abstract}

Key words: Pion-nucleus Optical Potential, $3 \alpha$-particle Model, $\Delta$-resonance Region

\section{INTRODUCTION}

${ }^{12} \mathrm{C}$ is a typical nucleus with the $\alpha$-particle structure. It is considered to consist of three $\alpha$-particles and these $\alpha$-particles basically retain the feature of a free $\alpha$-particle. The $\alpha$-particle is bounded much more weakly than a nucleon in the ${ }^{12} \mathrm{C}$ nucleus. Local $\pi$ nucleus optical potential was constructed based on the $\alpha$-particle model of the ${ }^{12} \mathrm{C}$ nucleus ${ }^{[1]}$ where the $\pi$ - $\alpha$ amplitude was directly obtained from fitting the experimental data. It was argued in Li Qing-run ${ }^{[1]}$ that the various effects indicated above would be automatically included to a certain extent in the $\pi-\alpha$ amplitudes. This simple model gave fairly good results over a wide energy region for $\pi-{ }^{12} \mathrm{C}$ elastic scattering, particularly in the low-energy region ${ }^{[1]}$.

Moreover, two forms of potential are commonly used to describe the pion-nucleus interaction. These two forms are the Kisslinger ${ }^{[2]}$ potential and a Laplacian ${ }^{[3]}$ one. Both contain explicit terms that originate in the pwave pion-nucleon interaction which are important near the $(3,3)$ resonance energy. The Kisslinger nonlocal potential $^{[2]}$ is:

$\mathrm{U}_{\mathrm{Kis}}(\mathrm{r})=\frac{(\hbar c)^{2}}{2 \omega}\{\mathrm{q}(\mathrm{r})+\nabla \cdot \alpha(\mathrm{r}) \nabla\}$

where, $\omega$ is the total energy of the pion in the center of mass (c.m.) system, the quantities $\mathrm{q}$ and $\alpha(\mathrm{r})$ mainly result from the s- and $\mathrm{p}$-waves of the pion-nucleon interaction and they are complex and energy dependent and given in detail by Johnson and Satchler ${ }^{[4]}$.

Recently, Johnson and Satchler ${ }^{[4]}$ used the KrellEricson transformation ${ }^{[5]}$, which leads from the KleinGordon equation for pion scattering to a local potential for the transformed wave function, equivalent to Kisslinger nonlocal potential. This local potential was used to successfully analyze the elastic scattering of $\pi^{ \pm}$ from ${ }^{12} \mathrm{C},{ }^{16} \mathrm{O},{ }^{28} \mathrm{Si}$ and ${ }^{40,44,48} \mathrm{Ca}$ in the pion kinetic energy range of 30 to $292 \mathrm{MeV}^{[6]}$. Elastic and inelastic scattering of positive and negative pions from calcium isotopes and ${ }^{54} \mathrm{Fe}$ were studied ${ }^{[7]}$ using the Kisslinger local potential, together with a zero-range DWBA code. The DWUCK4 code ${ }^{[8]}$ was used to calculate the differential cross-section angular distributions for elastically and inelastically scattered pions from these targets. It was concluded that the DWUCK4 code and the local-equivalent Kisslinger potential of Johnson and Satchler are reliable models for pion-nucleus scattering.

The aim of the present work is to calculate the angular distributions of the differential cross-sections of the $\pi^{ \pm}$elastically and inelastically scattered to the lowest $2^{+}$and $3^{-}$states in ${ }^{12} \mathrm{C}$ in the energy range of 50 to $260 \mathrm{MeV}$, using three independent methods of $\pi^{ \pm}$nucleus optical potential, the $3 \alpha$-particle model of the nucleus ${ }^{[1]}$, the local-equivalent Kisslinger potential ${ }^{[4]}$ and the Laplacian local potential ${ }^{[3]}$. The results of the three calculations are compared to the experimental data ${ }^{[9,10]}$. The total and reaction cross-section for these reactions are calculated and compared to the corresponding ones estimated by others.

\section{MATERIALS AND METHODS}

Three different forms of the optical potential have been used to study ion-nucleus interactions in the low and resonance regions. In the first form, the nuclear $\pi$ nucleus optical potential in the $\alpha$-particle model is local and given by Li Qing-run ${ }^{[1]}$ :

$\mathrm{U}_{00}(\mathrm{r})=\mathrm{B}_{0} \mathrm{Q}_{0}(\mathrm{r})+\mathrm{B}_{2} \mathrm{Q}_{2}(\mathrm{r})+\mathrm{B}_{4} \mathrm{Q}_{4}(\mathrm{r})+\mathrm{B}_{6} \mathrm{Q}_{6}(\mathrm{r})+\mathrm{B}_{8} \mathrm{Q}_{8}(\mathrm{r})$

where, the expressions for the B's and Q's are given in detail by Li Qing-run ${ }^{[1]}$. 
A second form of the pion-nucleus potential is obtained by the Kissinger local potential ${ }^{[4]}$, in this treatment the transformed potential is local and given by Johnson and Satchler ${ }^{[4]}$ :

$$
\begin{aligned}
& \mathrm{U}_{\mathrm{Loc}}(\mathrm{r})=\frac{(\hbar \mathrm{c})^{2}}{2 \omega}\left\{\frac{\mathrm{q}(\mathrm{r})}{1-\alpha(\mathrm{r})}-\frac{\mathrm{k}^{2} \alpha(\mathrm{r})}{1-\alpha(\mathrm{r})}-\frac{\frac{1}{2} \nabla^{2} \alpha(\mathrm{r})}{1-\alpha(\mathrm{r})}\right. \\
& \left.-\left(\frac{\frac{1}{2} \nabla \alpha(\mathrm{r})}{1-\alpha(\mathrm{r})}\right)^{2}\right\}+\frac{\alpha(\mathrm{r}) \mathrm{V}_{\mathrm{c}}}{1-\alpha(\mathrm{r})}
\end{aligned}
$$

with $\mathrm{q}(\mathrm{r})$ and $\alpha(\mathrm{r})$ the same as for the Kisslinger nonlocal potential and $\mathrm{K}$ the wave number of the pionnucleon in the center of momentum frame. The first term is nuclear local potential and the second term is the Coulomb correction. Here, the VC is the Coulomb potential due to the uniform charge distribution of the target nucleus of radius $R_{C}=r_{C} A^{1 / 3}$, $A$ is the target mass number and $r_{C}=1.2 \mathrm{fm}^{[7]}$.

A third method is the Laplacian model, where the potential is local and is written as ${ }^{[3]}$ :

$\mathrm{U}_{\mathrm{Lap}}=\frac{(\hbar \mathrm{c})^{2}}{2 \omega}\left\{\mathrm{q}(\mathrm{r})-\kappa^{2} \alpha(\mathrm{r})+\frac{1}{2} \nabla^{2} \alpha(\mathrm{r})\right\}$

$q(r)$ and $\alpha(r)$ are similar to those in the equation (1).

The first-order parameters $b_{i}$ and $c_{i}(i=0,1)$ for various pion kinetic energies $\mathrm{T} \pi$ considered here are calculated through the phase shifts, as they are computed in the code of Ebrahim and Peterson ${ }^{[11]}$. These parameters $b_{i}$ and $c_{i}$ are then used to generate the complex local potential $\mathrm{U}_{\mathrm{Loc}}$ using the expressions from Johnson and Satchler ${ }^{[4]}$. The same parameters $b_{i}$ and $c_{i}$ are also used for calculations in the Laplacian model to compare to the Kisslinger local potential calculations. The second-order parameters $\mathrm{B}_{0}$ and $\mathrm{C}_{0}$ which are very important at lower pion energies $\mathrm{T}<100 \mathrm{MeV}$ are taken into account here and are taken from Khallaf and Ebrahim $^{[6]}$. For inelastic $\pi$-nucleus scattering, the radial parts of the hadronic transition potentials used here are as follows ${ }^{[12]}$ :

$\mathrm{V}(\mathrm{r})=-\gamma_{\ell} \frac{\mathrm{dU}_{00}(\mathrm{r}) \text { or } \mathrm{dU}_{\mathrm{Loc}}(\mathrm{r}) \text { or } \mathrm{dU}_{\text {Lap }}(\mathrm{r})}{\mathrm{dr}}$

where, $\mathrm{U}_{00}$ is the pion-nucleus optical potential in the $\alpha$ particle model, $\mathrm{U}_{\mathrm{Loc}}(\mathrm{r})$ is the local transformed potential and $\mathrm{U}_{\mathrm{Lap}}(\mathrm{r})$ is the Laplacian potential. These potentials are those used to fit the corresponding elastic scattering data. In the present work, all other factors are kept the same as in case of elastic $\pi{ }^{12} \mathrm{C}$ scattering. When using the Kisslinger local or Laplacian potentials, the transformed wave function used in $\pi-{ }^{12} \mathrm{C}$ elastic scattering analysis is also employed in the case of inelastic scattering without any changes. For a given transition, we use $r_{l}$ to denote the corresponding "deformation lengths" for the $\pi^{ \pm}$interactions, where $l$ (= 2 or 3 ) is the multipolarity.

\section{RESULTS AND DISCUSSION}

To calculate the cross-sections for $\pi^{ \pm}$elastic scattering we have used the $\alpha$-particle model, the Kisslinger local potential and the Laplacian potential. The calculated results for ${ }^{12} \mathrm{C}$ involving no free parameters are compared with the experimental crosssections $^{[9,10]}$ in Fig. 1 and 3. For elastic and inelastic scattering from ${ }^{12} \mathrm{C}$, the values of the first- and secondorder parameters are of the same values for $\pi^{+}$and $\pi^{-}$ scattering at a certain beam energy. In the present calculations we have been using the three parameter Fermi shape of the density distributions of nucleons within ${ }^{12} \mathrm{C}$ along with the Ericson-Ericson LorentzLorentz (EELL) parameter $\zeta=1$. 0, they were more suitable for $\pi^{ \pm}$-nucleus scattering using the Kisslinger local potential ${ }^{[6]}$, in the same energy range considered here. To differentiate between the above-mentioned potentials, the quality of fits of the calculated differential cross-sections using these potentials are judged according to the $\chi^{2}$ - values.

We note that both $\alpha$-particle model and the Kisslinger local potential model give similar predictions except at large angles. In particular, both models predict two diffraction minima, but the predicted minima are much deeper than those observed. For Laplacian model, there exists a sizable discrepancy in the magnitude and shape of the cross-section and the calculated values does not have the energy dependence of the data.

In Fig. 1 the elastic scattering differential crosssections at forward angles and the positions of the minima and the maximum agree well with the calculated values of the Kisslinger local potential but at $260 \mathrm{MeV}$ calculations with the $\alpha$-particle model and Laplacian potential do not reproduce the depth and height of the structure around the minimum. Calculations using the first-order Kisslinger local potential are in better agreement with the experimental cross-sections at the three energies 150, 180 and 260 $\operatorname{MeV}\left(\chi^{2}=2.80-4.63\right)$ and $\alpha$-particle model calculations are in reasonable agreement with data $\left(\chi^{2}=9.13-11.65\right)$, while the Laplacian model calculations fail to agree the data three energies especial. ly at large angles $\left(\chi^{2}=10.41-14.12\right)$. The important difference between the Kisslinger local potential and Laplacian model is the charge effect, in the $U_{L o c}$ potential the $(1-\alpha(r))$ denominator applies also to the full local term, including the Coulomb term, while $\mathrm{U}_{\text {Lap }}$ do not have any Coulomb effects other than those explicitly in the transformed wave equation.

$$
\pi^{-}{ }_{-}{ }^{12} \mathrm{C} \text { complex Kisslinger local potential, }
$$

Laplacian model and $\alpha$-cluster model are shown in Fig. 2 at $180 \mathrm{MeV}$. 


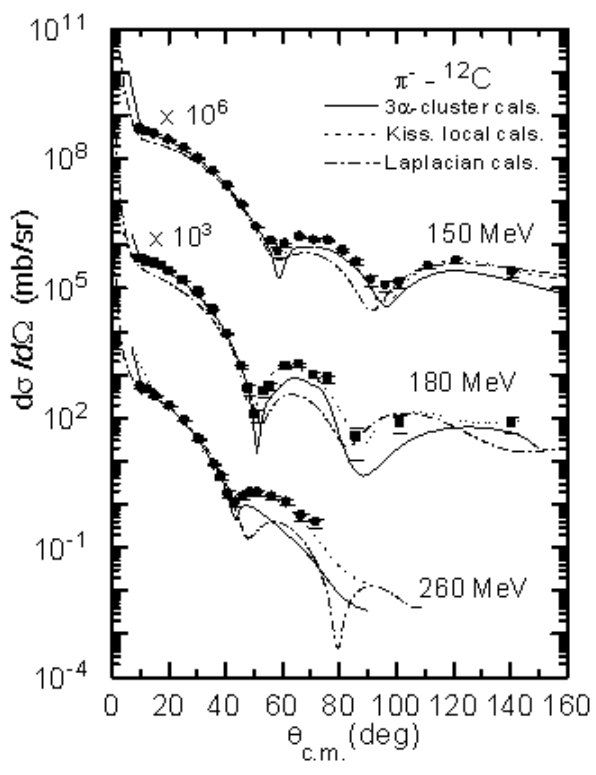

Fig. 1: Elastic Scattering Differential Cross-sections for 150,180 and $260 \mathrm{MeV} \pi^{+}$on ${ }^{12} \mathrm{C}$. Solid Points are the Experimental Data taken from Binon et al. ${ }^{[9]}$

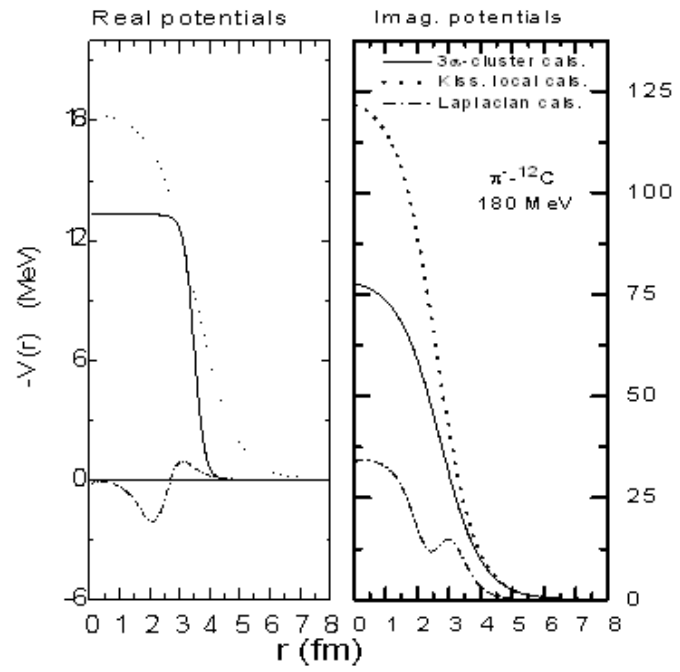

Fig. 2: Local Optical Potentials Computed for 180 $\mathrm{MeV} \pi \pi^{-}$Scattered from ${ }^{12} \mathrm{C}$

Both Kisslinger local potential and $3 \alpha$-cluster model are attractive for real and imaginary potentials, where the Kisslinger local potentials are deeper and wider while those of $\alpha$-particle model are shallower and sharper. For Laplacian model, the imaginary part is attractive while the real part is repulsive at smaller radii and attractive at large radii.

At lower pion beam energies $\mathrm{T}_{\pi}<100 \mathrm{MeV}$, the elastic scattering differential cross-sections of $\pi^{ \pm}$from ${ }^{12} \mathrm{C}$ of the pion kinetic energy $50 \mathrm{MeV}$ is calculated using the Three potential models.

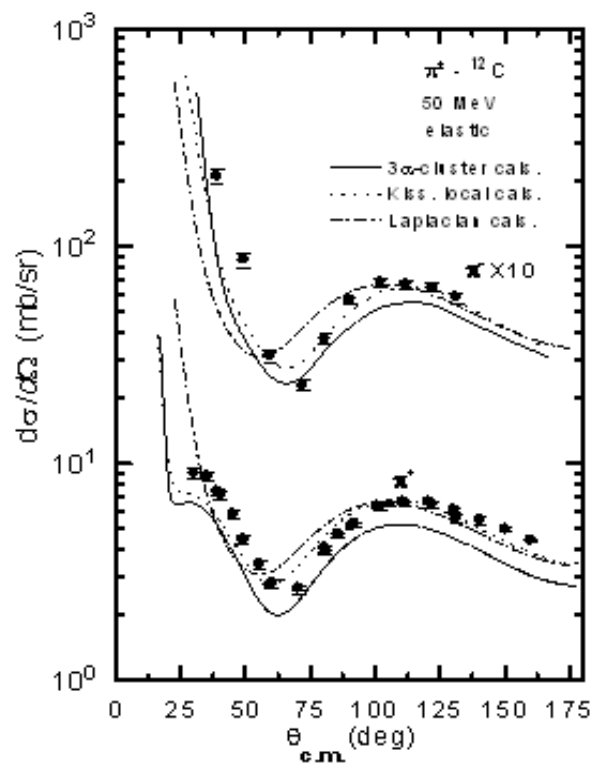

Fig. 3: As in Fig. 1 but for $\pi_{-}^{ \pm}{ }_{-}^{12}$ C Elastic Scattering Differential Cross-sections at $50 \mathrm{MeV}$ Pion Kinetic Energy. The Experimental Data are Taken from Sobie et al. ${ }^{[10]}$

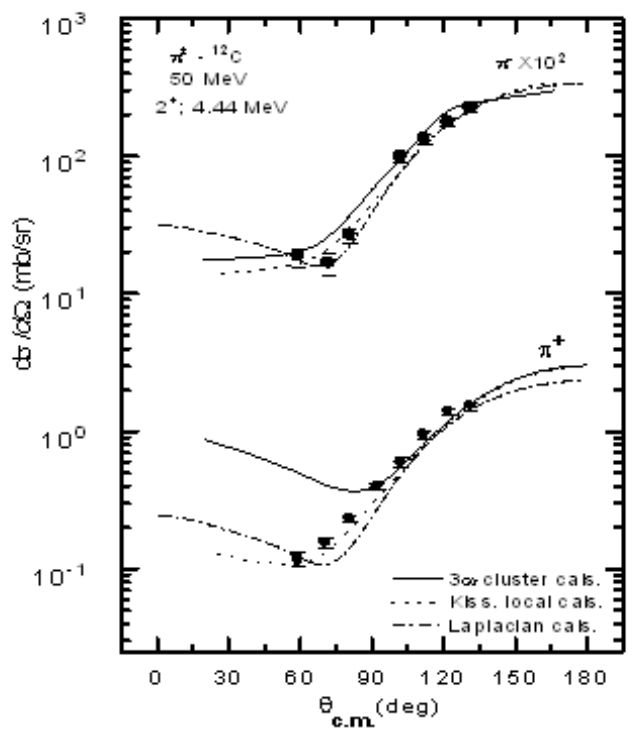

Fig. 4: As in Fig. 3 but for Inelastic Scattering Differential Cross-sections of $50 \quad \mathrm{MeV}^{ \pm}$ Exciting the $4.44 \mathrm{MeV} 2^{+}$State of ${ }^{12} \mathrm{C}$. The Experimental Data are taken from Sobie et al. ${ }^{[10]}$

Most of these calculations show non-negligible difference between the prediction of the differential cross-sections by the three potentials used here. We get a good agreement between the data and the Kisslinger local potential calculations when the second-order parameters are included beside the first-order parameters, as shown as dotted curves in Fig. 3 with $\chi^{2}=4.12$ for $\pi^{+}$and 3.44 for $\pi^{-}$. 
Table 1: Zero-energy pion $\quad{ }^{12} \mathrm{C}$ s-wave Scattering Lengths $\mathrm{a}_{0} \quad(\mathrm{fm})$ and $\mathrm{p}$-wave Scattering Volumes $a_{1}\left(\mathrm{fm}^{3}\right)$ Calculated using the Kisslinger Local and Laplacian Potentials Compared to Other Works ${ }^{[13]}$

\begin{tabular}{llll}
\hline & $\mathrm{U}_{\text {Loc }}$ cals. & $\mathrm{U}_{\text {Lop }}$ cals. & Others $^{[13]}$ \\
\hline $\mathrm{Rea}_{0}$ & -0.4398 & -0.4021 & $-0.438 \rightarrow 0.449$ \\
$\mathrm{Ima}_{0}$ & 0.1235 & 0.1182 & $0.122 \rightarrow 0.129$ \\
$\mathrm{Rea}_{1}$ & 1.8366 & 1.7815 & $1.88 \rightarrow 1.93$ \\
$\mathrm{Ima}_{1}$ & 0.4482 & 0.3517 & $0.347 \rightarrow 0.553$ \\
\hline
\end{tabular}

Calculations of the elastic scattering differential cross- section based on Laplacian model, included the second order interaction parameters, shown as dotdashed curves in Fig. 3 are in fair agreement with data for both $\pi^{+}\left(\chi^{2}=5.16\right)$ and $\pi^{-}\left(\chi^{2}=5.98\right)$. $\alpha$-particle optical potential calculations do rather poorly than those of the other two considered potentials for both $\pi^{+}\left(\chi^{2}=8.52\right)$ and $\pi^{-}\left(\chi^{2}=6.75\right)$.

As $\mathrm{k} \rightarrow 0$, the s-wave scattering length $\mathrm{a}_{0}=\delta_{0} / \mathrm{k}$ and p-wave scattering volume $a_{1}=\delta_{1} / \mathrm{k}^{3}$, where $\delta_{0}$ and $\delta_{1}$ are respectively the s- and p-wave phase shifts. Here $\mathrm{a}_{0}$ and $a_{1}$ are calculated at $1 \mathrm{KeV}$ for pions of both signs with the Coulomb potential omitted for the Kisslinger local and Laplacian Potentials ${ }^{[13]}$. The magnitude of (a) is a measure of the strength of the interaction and its sign indicates whether the interaction is effectively repulsive or attractive. The scattering lengths and volumes calculated from the two potentials are listed in Table 1 along with the values obtained from Stricker et al. ${ }^{[13]}$. Table 1 shows that magnitudes of $\mathrm{a}_{0}$ and $\mathrm{a}_{1}$ in the case of Kissinger local potential are greater than those of Laplacian potential. The quantities of $\mathrm{a}_{0}$ and $\mathrm{a}_{1}$ calculated here are in a good agreement both in sign and magnitude with those of Stricker et al. ${ }^{[13]}$.

Since inelastic scattering in the collective model is driven by the first derivative of the optical potential, agreement with such data can be a possible means to remove the ambiguity from elastic scattering fits. Here, angular distributions for the inelastic scattering of pions leading to the lowest $2^{+}$and $3^{-}$states in ${ }^{12} \mathrm{C}$ are computed by the DWBA method using the zero-range code DWUCK4 due to Kunz ${ }^{[8]}$. The $\alpha$-particle model optical potential observable, to predict observable of $\pi^{ \pm}$ inelastically scattered from nuclei. A collective model Distorted-wave Born Approximation (DWBA) prediction using the three potential models considered in the present work shows that the Kissinger local optical potential adequately fits the shape and magnitude of 50-260 MeV pion kinetic energies leading to the lowest $2^{+}$and $3^{-}$states in ${ }^{12} \mathrm{C}$ as shown in Fig. 46 . In the analysis presented here, the deformation lengths vary by agreement is obtained with $\pi^{ \pm}$data.

Figure 4 displays the predictions of the inelastic scattering differential cross-sections $\pi^{ \pm}$from ${ }^{12} \mathrm{C}$ nucleus excited for the lowest $2^{+}$state at $50 \mathrm{MeV}$.

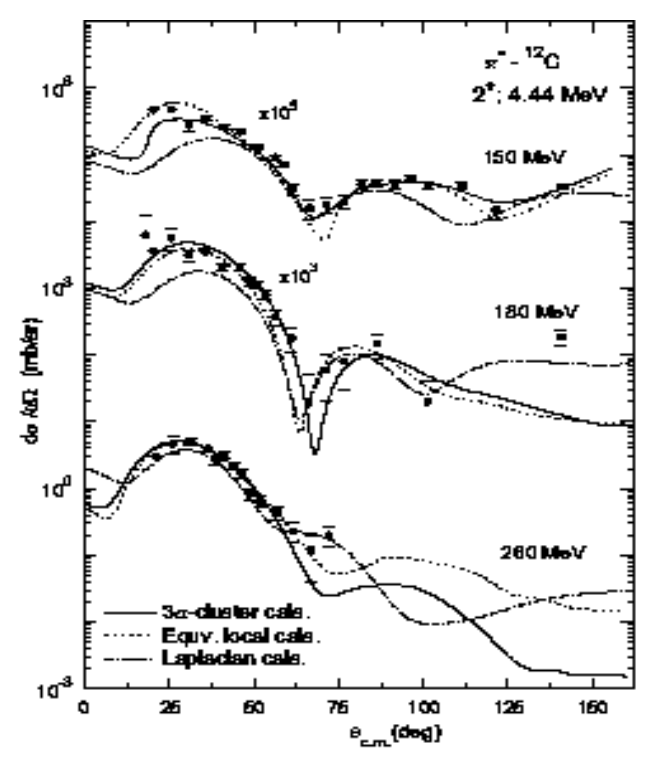

Fig. 5: As in Fig. 4, but for Inelastic Scattering Differential Cross-sections of 150, 180, 260 $\mathrm{MeV} \pi^{-}$Exciting the $4.44 \mathrm{MeV} 2^{+}$states of ${ }^{12} \mathrm{C}$. The Experimental Data are taken from Binon et al. ${ }^{[9]}$

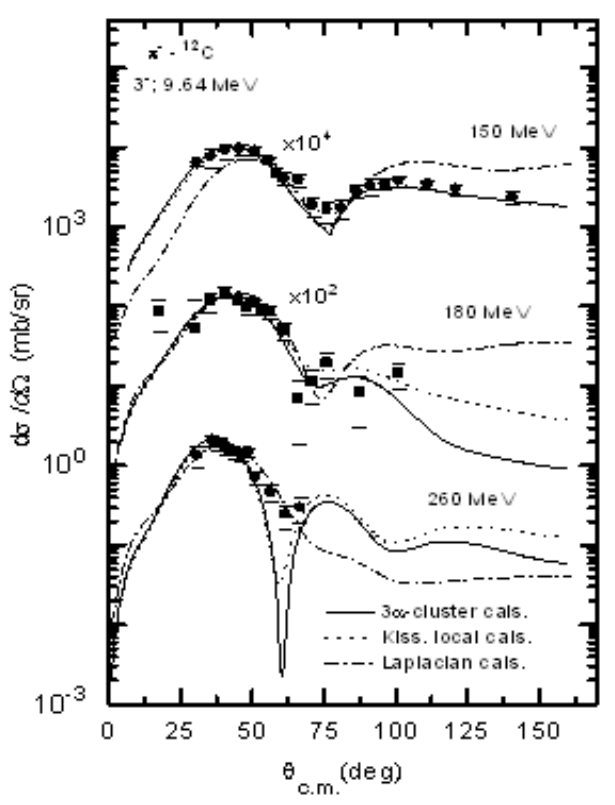

Fig. 6: As in Fig. 5, but for the $9.64 \mathrm{MeV} \mathrm{3}^{-}$State of ${ }^{12} \mathrm{C}$

The inelastic data ${ }^{[10]}$ are well represented by the present Kisslinger calculations with $\chi^{2}=1.82$ for $\pi^{+}$and 2.35 for $\pi^{-}$and Laplacian potential calculations with $\chi^{2}=4.28$ for $\pi^{+}$and 3.15 for $\pi^{-}$, when the first and second order parameters are included in both of potentials, while calculations based on the $3 \alpha$-particle model fail to agree with data at forward angles for $\pi^{+}$with $\chi^{2}=9.13$ and also give a poor fit with $\pi^{-}$data with $\chi^{2}=6.13$. 
Table 2: Deformation Lengths from $\pi^{ \pm}$Inelastic Scattering from ${ }^{12} \mathrm{C}$ Calculated using the Three Potential Methods Considered in the Present Work Compared to those Extracted from $\mathrm{K}^{+}$Inelastic Scattering and other Particles on ${ }^{12} \mathrm{C}^{[14-18]}$. The Corresponding $\chi^{2}$ values have also been calculated

\begin{tabular}{|c|c|c|c|c|c|c|c|c|}
\hline $\mathrm{T}_{\pi}(\mathrm{MeV})$ & 50 & 50 & 150 & 180 & 260 & 150 & 180 & 260 \\
\hline Pion & $\pi^{+}$ & $\pi^{-}$ & $\pi^{-}$ & & & & & \\
\hline State & $2^{+}$ & $3^{-}$ & & & & & & \\
\hline $3 \alpha$-cluster model $\gamma_{\text {real }}(\mathrm{fm})$ & 1.511 & 1.401 & 1.315 & 1.418 & 1.531 & 1.207 & 1.138 & 1.196 \\
\hline$\gamma_{\text {imag }}(\mathrm{fm})$ & 1.113 & 1.082 & 1.121 & 1.145 & 1.214 & 0.936 & 0.907 & 1.103 \\
\hline$\chi^{2}$ & 9.130 & 6.130 & 4.130 & 6.360 & 7.040 & 2.180 & 3.430 & 4.230 \\
\hline $\mathrm{U}_{\text {Loc }}$ model $\gamma_{\text {real }}(\mathrm{fm})$ & 1.514 & 1.412 & 1.405 & 1.517 & 1.604 & 1.273 & 1.212 & 1.277 \\
\hline$\gamma_{\text {imag }}(\mathrm{fm})$ & 1.213 & 1.103 & 1.207 & 1.243 & 1.316 & 1.025 & 0.982 & 1.112 \\
\hline$\chi^{2}$ & 1.820 & 2.350 & 5.050 & 5.880 & 6.210 & 3.630 & 3.080 & 4.190 \\
\hline $\mathrm{U}_{\text {Lap }}$ model $\gamma_{\text {real }}(\mathrm{fm})$ & 1.622 & 1.488 & 1.471 & 1.511 & 1.589 & 1.810 & 1.208 & 1.262 \\
\hline$\gamma_{\text {imag }}(\mathrm{fm})$ & 1.219 & 1.207 & 1.203 & 1.119 & 1.128 & 1.262 & 0.976 & 1.125 \\
\hline$\chi^{2}$ & 4.280 & 3.150 & 6.350 & 9.490 & 8.160 & 1.115 & 5.440 & 6.230 \\
\hline $\mathrm{K}^{+}$Scattering ${ }^{[14]} \gamma_{\text {real }}(\mathrm{fm})$ & $1.355-1.725$ & & & & & $1.302-1.512$ & & \\
\hline$\gamma_{\text {imag }}(\mathrm{fm})$ & $0.978-1.114$ & & & & & $0.955-1.214$ & & \\
\hline Others $\gamma(\mathrm{fm})$ & $1.12-1.97^{115]}$ & & $1.02-1.41^{[16]}$ & $1.07 \pm 0.05^{[17]}$ & $1.5-1.21^{[18]}$ & $0.65-1.23^{[15]}$ & & \\
\hline Reaction & $\begin{array}{l}\mathrm{p}-{ }^{12} \mathrm{C} \\
\mathrm{d}-{ }^{12} \mathrm{C} \\
{ }^{3} \mathrm{He}-{ }^{12} \mathrm{C}\end{array}$ & & ${ }^{3} \mathrm{He}^{-12} \mathrm{C}$ & $\alpha-{ }^{-12} \mathrm{C}$ & ${ }^{16} \mathrm{O}-{ }^{12} \mathrm{C}$ & $\begin{array}{l}{ }^{12} \mathrm{C}_{-}{ }^{12} \mathrm{C} \\
\alpha{ }^{-12} \mathrm{C} \\
{ }^{16} \mathrm{O}-{ }^{12} \mathrm{C}\end{array}$ & & \\
\hline
\end{tabular}

Table 3: Total and Reaction Cross-sections in $\mathrm{mb}$ for $\pi^{ \pm}$Scattering on ${ }^{12} \mathrm{C}$ Calculated in the Present Work Compared to Other Works

\begin{tabular}{|c|c|c|c|c|c|c|c|c|c|c|}
\hline \multirow{2}{*}{$\begin{array}{l}{ }_{\pi} \mathrm{T} \\
(\mathrm{MeV})\end{array}$} & \multirow[b]{2}{*}{ Pion } & \multicolumn{2}{|c|}{$3 \alpha$-cluster cals. } & \multicolumn{2}{|c|}{$\mathrm{U}_{\mathrm{Loc}}$ cals. } & \multicolumn{2}{|c|}{$\mathrm{U}_{\text {Lap }}$ model } & \multicolumn{3}{|l|}{ Others } \\
\hline & & $\sigma_{\mathrm{T}}$ & $\sigma_{R}$ & $\sigma_{\mathrm{T}}$ & $\sigma_{R}$ & & $\sigma_{\mathrm{T}}$ & $\sigma_{R}$ & $\sigma_{\mathrm{T}}$ & Refs. \\
\hline 50 & $\pi^{+}$ & 280.2 & 165.7 & 273.3 & 160.3 & 196.49 & 120.92 & 228 & 160 & [13] \\
\hline & & & & & & & & $248 \pm 20$ & $152 \pm 14$ & [19] \\
\hline 180 & & 590 & 388 & 570 & 380 & 641.66 & 422.26 & 581 & 384 & [13] \\
\hline 220 & & 532.3 & 322.54 & 530.36 & 313.72 & 550.51 & 336.62 & 521 & 318 & [13] \\
\hline 50 & $\pi^{-}$ & 302.1 & 190.8 & 292.7 & 189.15 & 258.74 & 153.19 & 290 & 200 & [13] \\
\hline 150 & & 703.3 & 467.63 & 683.5 & 442.8 & 703.25 & 467.63 & $696 \pm 7$ & & [9] \\
\hline 180 & & 675.03 & 439.97 & 666.2 & 424.1 & 675.33 & 439.25 & $\begin{array}{l}615 \\
670+7\end{array}$ & 400 & [13] \\
\hline 220 & & 612.19 & 343.14 & 590.23 & 340.13 & 578.72 & 349.41 & $\begin{array}{l}552 \\
586\end{array}$ & 330 & [1] \\
\hline 260 & & 499.1 & 325.4 & 529.4 & 315.6 & 487.62 & 277.27 & $536 \pm 6$ & & [9] \\
\hline
\end{tabular}

Figure 5 and 6 display the predictions of the inelastic scattering differential cross-sections of $\pi^{-}$from ${ }^{12} \mathrm{C}$ nucleus excited to the lowest $2^{+}$and $3^{+}$states in 150,180 and $260 \mathrm{MeV}$. The three forms for the optical potential models give reasonable agreement with inelastic scattering data of ${ }^{99]}$, but the Kisslinger local potential predictions seem to be better at all energies considered in the present work with $\chi^{2}$ ranging from 5.05-6.21 for $2^{+}$and 1.81-4.19 for $3^{-}$states. These values of $\chi^{2}$ are larger for Laplacian model $\left(\chi^{2}=6\right.$. 358.16) for $2^{+}$and $\left(\chi^{2}=3\right.$. 63-6.23) for $3^{-}$states, while for $\alpha$-particle model $\left(\chi^{2}=4\right.$. 13-7.04) for $2^{+}$and $\left(\chi^{2}=2\right.$. 184.23) for $3^{-}$states except the case of $150 \mathrm{MeV} \pi^{-}$ inelastic scattering.

From the above, we note that the fits reproduced on the basis of the $\alpha$-cluster model of ${ }^{12} \mathrm{C}$ nucleus are more reasonable for low energy pions than for pions of higher energies. This is consistent with the conclusions of Li Qing-run ${ }^{[1]}$. This may indicate that the clustering phenomena in ${ }^{12} \mathrm{C}$ nucleus are more dominant for low pion energy scattering while pions of higher energies prefer to interact with the $12 \mathrm{C}$ nucleus as a whole. The predictions of the Kisslinger local potential will fit the data for differential cross-sections at all energies under consideration.

The values of the deformation lengths for all collective states analyzed here are summarized in Table 2 and compared to those extracted from $\mathrm{K}^{+}$scattering ${ }^{[14]}$ and to the corresponding ones previously extracted by others $^{[15-18]}$. It is clear from Table 2 that the deformation lengths of the real potential are greater than the corresponding ones for the imaginary potential in all cases under consideration. Real deformation lengths extracted here at $150 \mathrm{MeV}$ for $2^{+}$and $180 \mathrm{MeV}$ since $3^{-}$ 
are minimized. All values of deformation lengths extracted from the present work lie within or very close to the range of the corresponding values previously extracted from $\mathrm{K}^{+}$scattering and other particles on ${ }^{12} \mathrm{C}^{[14-18]}$. It can be seen from Table 2 that the values of imaginary deformation lengths determined here using the 3 $\alpha$-particle model and Kisslinger local potential for $2^{+}$and $3^{-}$excited states in the $12 \mathrm{C}$ increase with increasing pion kinetic energy, except for the case of $180 \mathrm{MeV} \pi^{-}$ inelastic scattering of $3^{-}$state in ${ }^{12} \mathrm{C}$. This $180 \mathrm{MeV}$ energy lies in the $(3,3)$ resonance region of pions. Deformation length values extracted from the Kisslinger local and Laplacian potentials are higher than those extracted from the 3 $\alpha$-particle model, except for the imaginary deformation lengths for 180 and 260 $\mathrm{MeV} \pi^{-}$off $2^{+}$state in ${ }^{12} \mathrm{C}$ using the Laplacian potential. Table 2 also includes the calculated $\chi^{2}$ values corresponding to each case under consideration. Again, it shows that $\chi^{2}$ is minimum for the local Kisslinger potential in each of these cases except for $\pi^{-}$inelastic scattering of $150 \mathrm{MeV}$ kinetic energy scattered to $2^{+}$ and $3^{-}$excited states in ${ }^{12} \mathrm{C}$.

The DWUCK4 code using either of the three forms of potential considered here calculates the reaction cross- sections $\sigma_{\mathrm{R}}$ of pion scattering from ${ }^{12} \mathrm{C}$ at pion kinetic energy ranging from 50 to $260 \mathrm{MeV}$. Table 3 shows the predicted $\sigma_{\mathrm{R}}$ and $\sigma_{\mathrm{T}}$ for pions of both signs scattering on ${ }^{12} \mathrm{C}$ at $50-260 \mathrm{MeV}$ pion kinetic energy together with the corresponding cross-sections estimated by others. The values of $\sigma_{\mathrm{R}}$ and $\sigma_{\mathrm{T}}$ predicted by these three potentials were found to be the same to within $1 \%$ regardless of the potential used but in most cases the local Kisslinger potential predictions are the nearest to the corresponding cross-sections estimated by others. This result is not surprising in view of the short mean-free path of pions in nuclei in this energy range which should make most of the scattering takes place in the nuclear surface. This is in contrast to the situation with low-energy pions. From Table 3 with the three forms of potential models, it is noticed for $\pi^{ \pm}$scattering of $\mathrm{T} \geq 180 \mathrm{MeV}$ from ${ }^{12} \mathrm{C}$ that both calculated $\sigma_{\mathrm{R}}$ and $\sigma_{\mathrm{T}}$ decrease as the beam energy increases and at all considered energy values of $\sigma_{\mathrm{T}}$ for $\pi^{-}$are greater than those for $\pi^{+}$at a certain energy. This indicates that the mean free path $\lambda$ for $\pi^{-}$is shorter than the corresponding $\lambda$ for $\pi^{+}$. This is consistent with our previous results ${ }^{[7]}$.

\section{CONCLUSION}

Elastic, inelastic, total and reaction cross-sections are calculated using DWBA and the three forms of potential models, Kisslinger local, Laplacian and the $3 \alpha$-particle model formalisms. For the elastic scattering, there is a noticeable disagreement between the $3 \alpha-$ particle model calculations and data, especially at large angles in the region of $(3,3)$ resonance; the data are larger than theory by a large factor. In the case of inelastic scattering, relatively little difference is seen between data and the $\alpha$-particle model calculations. As the energy decreases the agreement with experiment gets better.

We are able to obtain a good fit to the data for the elastic and inelastic scattering of 50-260 MeV pions from ${ }^{12} \mathrm{C}$, using the Kisslinger local potential. The Kisslinger local potential calculations are much more comprehensive than $\alpha$-particle model and Laplacian potential treatments, we may say that Johnson and Satchler emphasize a careful treatment of the first and second order optical potential. That potential includes also short-range correlations $\zeta$. The second order parameters will be necessary to explain the data at lower pion energies $<100 \mathrm{MeV}$. The mean free path $\lambda$ for $\pi^{-}$is shorter than the corresponding $\lambda$ for $\pi^{+}$.

\section{REFERENCES}

1. Li Qing-run, 1984. Pion-nucleus optical potential in the $\alpha$-particle model. Nucl. Phys., A415: 445.

2. Kisslinger, L.S., 1955. Scattering of mesons by light nuclei. Phys. Rev., 98: 761.

3. Göran Fäldt; 1972. On the pion-nucleus interaction in the (3,3) -resonance region. Phys. Rev., C5: 400.

4. Johnson, M.B. and G.R. Satchler; 1996. Characteristics of local pion-nucleus potentials that are equivalent to Kisslinger-type potentials. Ann. Phys., N.Y., 248: 134.

5. Krell, M. And T.E.O. Ericson, 1969. Energy levels and wave functions of pionic atoms. Nucl. Phys., B11: 521.

6. Khallaf, S.A.E. and A.A. Ebrahim, 2000. Analysis of $\pi^{ \pm}$-nucleus elastic scattering using a local potential. Phys. Rev., C62: 024603.

7. Khallaf, S.A.E. and A.A. Ebrahim, 2002. Elastic and inelastic scattering of pions from nuclei using an equivalent local potential. Phys. Rev., C65: 064605.

8. Kunz. P.D. Zero Range Distorted Wave Born Approximation. DWBA code DWUCK4 University of Colorado (unpublished).

9. Binon, F., P. Duteil, J.P. Garron, J. Gorres, L. Hugon, J. P .Peigneux, C. Schmit, M. Spighel and J.P. Stroot, 1970. Scattering of negative pions on carbon. Nucl. Phys., B17: 168.

10. Sobie, R.J. et al., 1984. Elastic and inelastic scattering of $50 \mathrm{MeV}$ pions from ${ }^{12} \mathrm{C},{ }^{32} \mathrm{~S}$ and ${ }^{34} \mathrm{~S}$. Phys. Rev., C30: 1612.

11. Ebrahim, A.A. and R.J. Peterson, 1996. Parameterization of pion-nucleus phase shifts and effects upon pion-nucleus scattering calculations. Phys. Rev., C54: 2499. 
12. Satchler, G.R., 1992. A local potential model for pion-nucleus scattering and $\pi^{+} / \pi^{-}$excitation ratios. Nucl. Phys., A540: 533.

13. Stricker, K., H. McManus and J.A. Carr, 1979. Nuclear scattering of low energy pions. Phys. Rev., C19: 929.

14. Ebrahim, A.A. and S.A.E. Khallaf, 2002. Elastic and inelastic scattering of $\mathrm{K}^{+}$from nuclei. Phys. Rev., C66: 044614.

15. Smith, S.M., G. Tibell, A.A. Cowley, D.A. Goldberg, H.G. Pugh, W. Reichart and N.S. Wall, 1973. The $(\alpha, \alpha),\left(\alpha, \alpha^{\prime}\right)$ and $\left(\alpha,{ }^{3} \mathrm{He}\right)$ reactions on ${ }^{12} \mathrm{C}$ at $139 \mathrm{MeV}$. Nucl. Phys., A207: 273.

16. Dem' Yanova, A.S., E.F. Svinareva, S.A. Goncharov, S.N. Ershov, F.A. Gareev, G.S. Kazacha, A.A. Ogloblin and J.S. Vaagen, 1992. Scattering of ${ }^{3} \mathrm{He}$ on ${ }^{12} \mathrm{C}$ and the inelastic form factor. Nucl. Phys., A542: 208.
17. Brandan, M.E. and K.W. McVoy, 1991. Rainbowshift mechanism behind discrete optical-potential ambiguities. Phys. Rev., C43: 1140.

18. Moffa, P.J., C.B. Dover and J.P. Vary, 1977. Folding model description of heavy ion inelastic scattering. Phys. Rev., C16: 1857.

19. Saunders, A., S. HØibråten, J.J. Kraushaar, B.J. Kriss, R.J. Peterson, R.A. Ristinen, J.T. Brack, G. Hofman, E.F. Gibson and C.L. Morris, 1996. Reaction and total cross-sections for low energy $\pi^{+}$ and $\pi^{-}$on isospin zero nuclei. Phys. Rev., C53: 1745 . 